Research Paper

\title{
Acute Alcohol Intoxication Exacerbates Rhabdomyolysis-Induced Acute Renal Failure in Rats
}

\author{
Jen-Pi Tsai 1, 2, Chung-Jen Lee 3, Yi-Maun Subeq ${ }^{4}$, Ru-Ping Lee 5, Bang-Gee Hsu 2, $6 \bowtie$ \\ 1. Division of Nephrology, Department of Internal Medicine, Dalin Tzu Chi Hospital, Buddhist Tzu Chi Medical Foundation, Chiayi, Taiwan; \\ 2. School of Medicine, Tzu Chi University, Hualien, Taiwan; \\ 3. Department of Nursing, Tzu Chi University of Science and Technology, Hualien, Taiwan; \\ 4. Department of Nursing, Tzu Chi University, Hualien, Taiwan; \\ 5. Institute of Medical Sciences, Tzu Chi University, Hualien, Taiwan; \\ 6. Department of Nephrology, Tzu Chi General Hospital, Hualien, Taiwan. \\ $\square$ Corresponding author: gee.lily@msa.hinet.net; Tel.: +886-3-8561825
}

(c) Ivyspring International Publisher. This is an open access article distributed under the terms of the Creative Commons Attribution (CC BY-NC) license (https://creativecommons.org/licenses/by-nc/4.0/). See http://ivyspring.com/terms for full terms and conditions.

Received: 2017.02.03; Accepted: 2017.04.20; Published: 2017.06.23

\begin{abstract}
Traumatic and nontraumatic rhabdomyolysis can lead to acute renal failure (ARF), and acute alcohol intoxication can lead to multiple abnormalities of the renal tubules. We examined the effect of acute alcohol intoxication in a rat model of rhabdomyolysis and ARF. Intravenous injections of $5 \mathrm{~g} / \mathrm{kg}$ ethanol were given to rats over $3 \mathrm{~h}$, followed by glycerol-induced rhabdomyolysis. Biochemical parameters, including blood urea nitrogen (BUN), creatinine (Cre), glutamic oxaloacetic transaminase (GOT), glutamic pyruvic transaminase (GPT), and creatine phosphokinase (CPK), were measured before and after induction of rhabdomyolysis. Renal tissue injury score, renal tubular cell expression of E-cadherin, nuclear factor-KB (NF-KB), and inducible nitric oxide synthase (iNOS) were determined. Relative to rats in the vehicle group, rats in the glycerol-induced rhabdomyolysis group had significantly increased serum levels of BUN, Cre, GOT, GPT, and CPK, elevated renal tissue injury scores, increased expression of NF-KB and iNOS, and decreased expression of E-cadherin. Ethanol exacerbated all of these pathological responses. Our results suggest that acute alcohol intoxication exacerbates rhabdomyolysis-induced ARF through its pro-oxidant and inflammatory effects.
\end{abstract}

Key words: Acute renal failure; Acute ethanol intoxication; Rhabdomyolysis.

\section{Introduction}

There are multiple causes of rhabdomyolysis, which is defined by the breakdown and necrosis of striated muscles, and the leakage of myoglobin, electrolytes, and other sarcoplasmic proteins into the urine. These include traumatic injury, hyperthermia, infection, electrolyte imbalances, toxins, and certain medications $[1,2]$. The severity of injuries caused by rhabdomyolysis range from asymptomatic elevation of serum creatine kinase (CK) to life-threatening conditions, such as disseminated intravascular coagulation, and acute renal failure (ARF) [3]. Irrespective of its cause, $5-25 \%$ of rhabdomyolysis cases experience ARF, and such patients have an increased risk of mortality $[1,4]$.

Previous research identified several possible mechanisms of rhabdomyolysis-induced ARF, including vasoconstriction, formation of intra-tubular casts, and a direct toxic effect of myoglobin on renal tubular cells $[5,6]$. The heme cofactors released from myoglobin can produce reactive oxygen species (ROS), scavenge nitric oxide, and activate endothelin receptors, which interact synergistically to cause renal vasoconstriction and intra-tubular cast formation, and ultimately lead to myoglobinuric $\operatorname{ARF}[7,8]$. The treatments for rhabdomyolysis-induced ARF include aggressive hydration, mannitol administration, urine alkalization, as well as several recent promising strategies that seek to lessen vasoconstriction, to increase the levels of anti-oxidants, and to inhibit inflammation $[8,9]$. These new strategies highlight the 
important roles of pro-oxidants and inflammatory injuries in the pathogenesis of rhabdomyolysisinduced ARF.

Acute alcohol intoxication is a clinically harmful condition that can cause traumatic injuries, negatively affect the function of multiple organs, and lead to increased rates of morbidity and mortality [10, 11]. Although moderate alcohol consumption is cardio-protective, heavy alcohol consumption increases the risk of renal disease because it negatively affects endothelial function, which increases the risk for renal damage [12, 13]. Thus, alcohol-fed rats develop significant renal dysfunction, interstitial edema, and renal hypertrophy [14], and increased levels of oxidized proteins and lipids in the kidneys [13, 15]. A recent study reported that rats given intravenous ethanol before induction of hemorrhagic shock had greatly aggravated renal tubular necrosis scores and elevated serum levels of pro-inflammatory cytokines [11]. Rhabdomyolysis can both cause renal damage. The present study examines whether acute alcohol intoxication aggravates organ dysfunction, and especially renal dysfunction, after glycerol-induced rhabdomyolysis.

\section{Materials and Methods}

\section{Preparation of animals}

Thirty-two male Sprague-Dawley rats, weighing 280-300 g, were purchased from the National Animal Center (Taipei, Taiwan). The rats were housed in our animal center under a controlled environment at a temperature of $22 \pm 1^{\circ} \mathrm{C}$ with a 12 hour light/dark cycle. Food and water were provided ad libitum. The experimental protocol was approved by the Animal Usage Regulation Committee of Tzu Chi Hospital.

The animals were anesthetized by ether inhalation for about $10 \mathrm{~min}$. During anesthesia, polyethylene catheters (PE-50) were inserted into the femoral artery for collection of blood samples and the femoral vein for intravenous administration of drugs or fluids. The operation was completed within $15 \mathrm{~min}$, and the wound was kept as small as possible (less than $0.5 \mathrm{~cm}^{2}$ ). After the operation, animals were placed in conscious rat metabolic cages (Shingshieying Instruments, Hualien, Taiwan). The rats were awakened soon after the operations, and rhabdomyolysis was induced by glycerol injection 24 $\mathrm{h}$ later while they were conscious, as previously described [11, 16, 17].

\section{Induction of acute alcohol intoxication}

Twenty-four hours after insertion of the PE-50 catheters, acute alcohol intoxication was induced by administration of intravenous ethanol $(5 \mathrm{~g} / \mathrm{kg})$ in normal saline (total volume: $4 \mathrm{~mL}$ ) over $3 \mathrm{~h}$ [11].

\section{Experimental design}

The 32 animals were randomly divided into four groups, with 8 rats per group: vehicle group, glycerol group, ethanol group, and ethanol + glycerol group. Rats in the vehicle group and glycerol group were given intravenous normal saline $(4 \mathrm{~mL})$ over $3 \mathrm{~h}$, and then intramuscular normal saline (vehicle group; 10 $\mathrm{mL} / \mathrm{kg}$ saline) or saline with $50 \%$ glycerol (glycerol group; $10 \mathrm{~mL} / \mathrm{kg}$; Sigma Chemical, St. Louis, MO, USA) in each hind leg. Rats in the ethanol group and the ethanol + glycerol group were given intravenous ethanol $(5 \mathrm{~g} / \mathrm{kg})$ in normal saline (total volume: $4 \mathrm{~mL}$ ) over $3 \mathrm{~h}$, and then intramuscular normal saline (ethanol group; $10 \mathrm{~mL} / \mathrm{kg}$ saline) or saline with $50 \%$ glycerol (ethanol + glycerol group) in each hind leg. Rats were sacrificed by decapitation $48 \mathrm{~h}$ after glycerol administration $[16,17]$.

\section{Biochemical analyses}

Blood samples $(0.5 \mathrm{~mL})$ for measurements of BUN, Cre, GOT, GPT, and CPK were taken before and 1 to $48 \mathrm{~h}$ after glycerol administration. These samples were immediately centrifuged at $3000 \mathrm{~g}$ for $10 \mathrm{~min}$, decanted, and then stored at $4^{\circ} \mathrm{C}$. Biochemical analyses were performed within $1 \mathrm{~h}$ of collection. The serum levels of all analytes were measured using an autoanalyzer (COBAS C111, Roche Diagnostics, Basel, Switzerland) [11, 16, 17]

\section{Histo-pathological examination}

The kidneys were removed immediately after sacrifice. Tissue specimens were fixed overnight in $4 \%$ buffered formaldehyde, processed by standard methods, and stained with hematoxylin and eosin (H\&E). The observer who examined the tissue specimens was blinded to the experimental groups. The renal tissue injury score was determined by the percentage of tubules in the cortex or the outer medulla that exhibited epithelial necrosis, luminal necrotic debris, tubular dilation, and development of heme casts: 0 , none; $1,<5 \% ; 2,5 \%$ to $<25 \% ; 3,25 \%$ to $75 \%$; and $4,>75 \%[16,17]$. There were 5 sections per kidney, and 5 fields per section were examined.

\section{Immunohistochemical (IHC) staining}

For IHC staining, serial $4-\mu \mathrm{m}$ sections were deparaffinized, rehydrated, and incubated with different mouse monoclonal antibodies at $4^{\circ} \mathrm{C}$ overnight, according to the manufacturer's directions. Antigen retrieval was performed for E-cadherin (\#E0411), nuclear factor-kB/P65 (NF-kB p65) (\#I0712), and iNOS (\#110420DA) at dilutions of 1:150 (Neomarkers, Lab Vision Corporation, Fremont, California, USA). After incubation, tissue sections were covered with a biotinylated goat anti-mouse 
polyvalent secondary antibody, and incubated at room temperature for $10 \mathrm{~min}$. The slides were washed, and then incubated in a peroxidase conjugated streptavidin-biotin complex (Dako, Copenhagen, Denmark) for $10 \mathrm{~min}$. Cells positive for each protein were semi-quantitatively determined from paraffin-embedded tissue sections by examination of 10 high-power fields (200×) per section. Data are expressed as the percentage of positive staining of the total area examined [16, 17]. All scoring was performed in a blinded manner using coded slides.

\section{Statistical analysis}

Data are expressed as means \pm SEMs. Statistical comparisons between different groups at different time points were performed using a repeated measures two-way analysis of variance, followed by a post hoc test (Bonferroni's method). Histological scores were analyzed using the Kruskal-Wallis test or the Mann-Whitney $U$ test, as appropriate. A $p$ value less than 0.05 was considered statistically significant.

\section{Results}

\section{Creatine phosphokinase (CPK), Glutamic oxaloacetic transaminase (GOT) and glutamic pyruvic transaminase (GPT)}

Rats that were given glycerol to induce rhabdomyolysis had maximal levels of CPK, GOT, and GPT at $6 \mathrm{~h}$, and the levels of these markers were significantly greater than those of rats given vehicle alone from $1 \mathrm{~h}$ to $48 \mathrm{~h}(P<0.05$; Fig. $1 \mathrm{~A}, 1 \mathrm{~B}$, and $1 \mathrm{C})$. Relative to the glycerol group, rats in the ethanol + glycerol group had greater serum levels of CPK at $1 \mathrm{~h}$ and $3 \mathrm{~h}$, and greater levels of GOT and GPT at $6 \mathrm{~h}$ to 48 h $(P<0.05$; Fig. 1A, 1B, 1C).

\section{Serum blood urea nitrogen (BUN) and creatinine (Cre)}

Rats in the glycerol group had greater serum levels of BUN and Cre from $1 \mathrm{~h}$ to $48 \mathrm{~h}$ relative to the vehicle group $(P<0.05 ;$ Fig. $1 \mathrm{D}$, and $1 \mathrm{E})$. In addition, relative to the glycerol group, rats in the ethanol + glycerol group had greater serum levels of BUN and Cre at all time points $(P<0.05$; Fig. $1 \mathrm{D}$ and 1E).

\section{Renal histo-pathological examinations}

Histological analysis of the kidneys of rats in the vehicle and ethanol groups indicated no epithelial necrosis, and no heme casts (Fig. 2B and 2C). However, the kidneys of rats in the glycerol group and the ethanol + glycerol group had moderate epithelial necrosis, tubular dilation, and several heme casts due to rhabdomyolysis (Fig. 2A and 2D).
The renal tissue injury scores were significantly greater for rats in the glycerol group than the vehicle group at $48 \mathrm{~h}$ after induction of rhabdomyolysis $(P<$ 0.05 ; Fig. 2E). In addition, the renal tissue injury scores were significantly greater for rats in the ethanol + glycerol group relative to the glycerol group at $48 \mathrm{~h}(P$ $<0.05$; Fig. 2E).

\section{Renal Immunohistochemical staining of E-cadherin}

IHC staining of renal tissues indicated that rats in the vehicle and ethanol groups had normal amounts and distributions of renal tubular E-cadherin (Fig. 3B and 3C). However, the renal tissues of rats in the glycerol group and the ethanol + glycerol group had significantly decreased renal tubular expression of E-cadherin (Fig. 3A and 3D).

Semi-quantitative analysis indicated rats in the glycerol had group significantly decreased expression of renal tubular E-cadherin relative to the vehicle group. In addition, rats in the ethanol + glycerol group had lower expression of renal tubular E-cadherin than rats in the glycerol group $(P<0.05$; Fig. 3E).

\section{Renal Immunohistochemical staining of iNOS and NF-KB}

IHC staining of renal tissues indicated that rats in the vehicle and ethanol groups had normal amounts and distributions of renal tubular iNOS and NF-kB (Fig. 4B, 4C, 5B, and 5C). However, rats in the glycerol group and the ethanol + glycerol group had significantly increased renal tubular expression of iNOS and NF-kB (Fig. 4A, 4D, 5A, and 5D).

Semi-quantitative analysis indicated significantly increased expression of iNOS and NF-kB in the glycerol group relative to the vehicle group, and significantly greater expression of iNOS and NF-KB in the ethanol + glycerol group relative to the glycerol group $(P<0.05$; Fig. 4E and $5 \mathrm{E})$.

\section{Discussion}

The major finding of this study of a rat model of rhabdomyolysis is that heavy alcohol intoxication before glycerol-induced rhabdomyolysis exacerbates organ damage, increases renal tubular expression of NF-kB and iNOS, and decreases expression of E-cadherin.

Rhabdomyolysis is a condition that occurs following muscle damage, in which large quantities of potassium, phosphate, CK, GOT, urate, and myoblobin are released into the circulation $[1-3,18]$. The main causes of rhabdomyolysis in humans are toxin-induced damage of cell membranes, crash injuries, and inadequate ATP supply following 
ischemia. Any of these can induce a pathologic interaction of actin and myosin, resulting in activation of intra-cellular proteases that destroy muscle fibers, and ultimately lead to necrosis [19]. After rhabdomyolysis, ARF develops in up to $15 \%$ of patients, and there are associated increases in morbidity and mortality [20]. In this study of a rat model of rhabdomyolysis, we found that rhabdomyolysis increased serum and pathological markers of renal injury, including BUN, Cre, and renal tubular necrosis score.
(A)

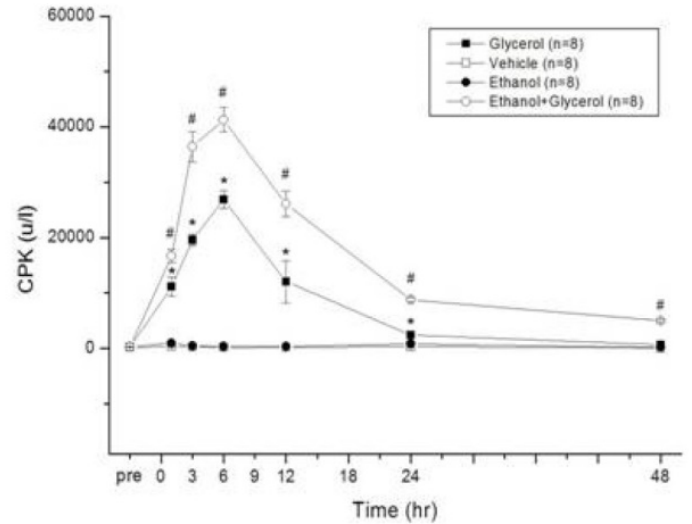

(C)

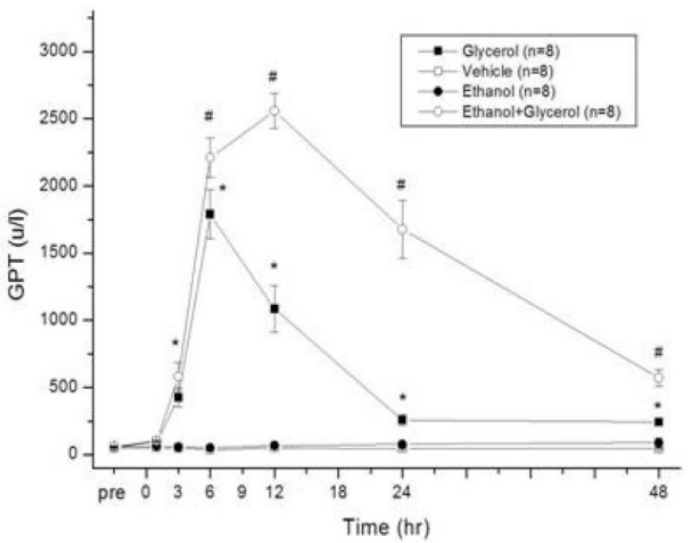

(E)

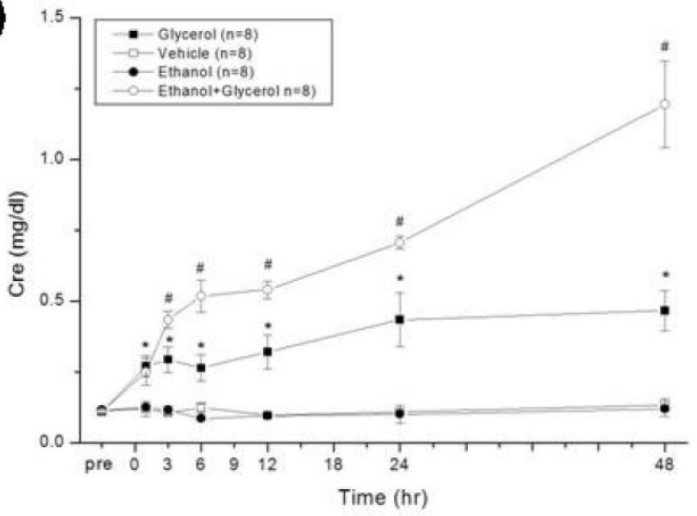

(B)

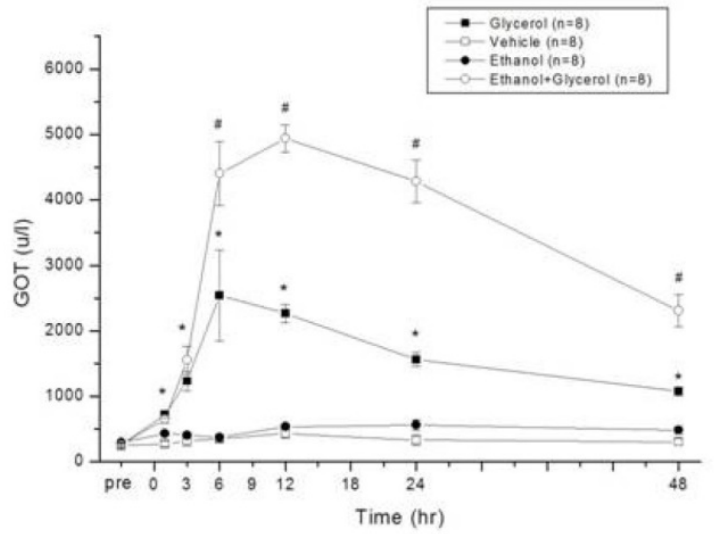

(D)

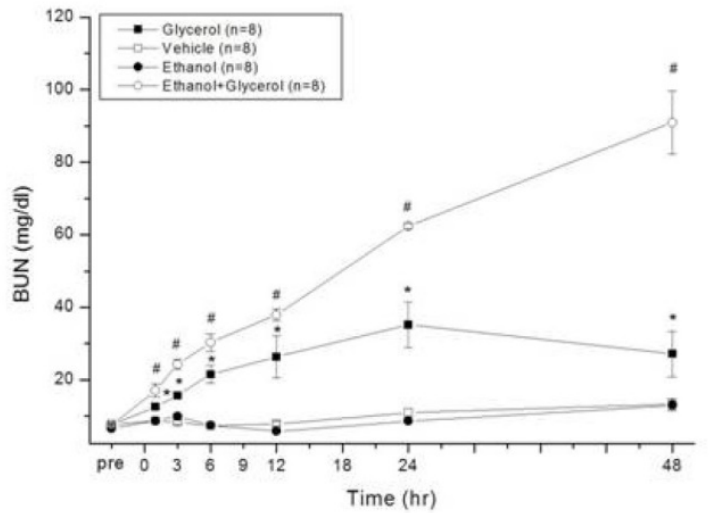

Figure 1. Change in creatine phosphokinase (CPK) (A), glutamic oxaloacetic transaminase (GOT) (B), glutamic pyruvic transaminase (GPT) (C), blood urea nitrogen (BUN) (D) and creatinine (Cre) (E) after rhabdomyolysis-induced acute renal failure in rats. ${ }^{*} p<0.05$ for the Glycerol group compared with the Vehicle group. ${ }^{*} p<$ 0.05 for the Ethanol + Glycerol group compared with the Glycerol group. 

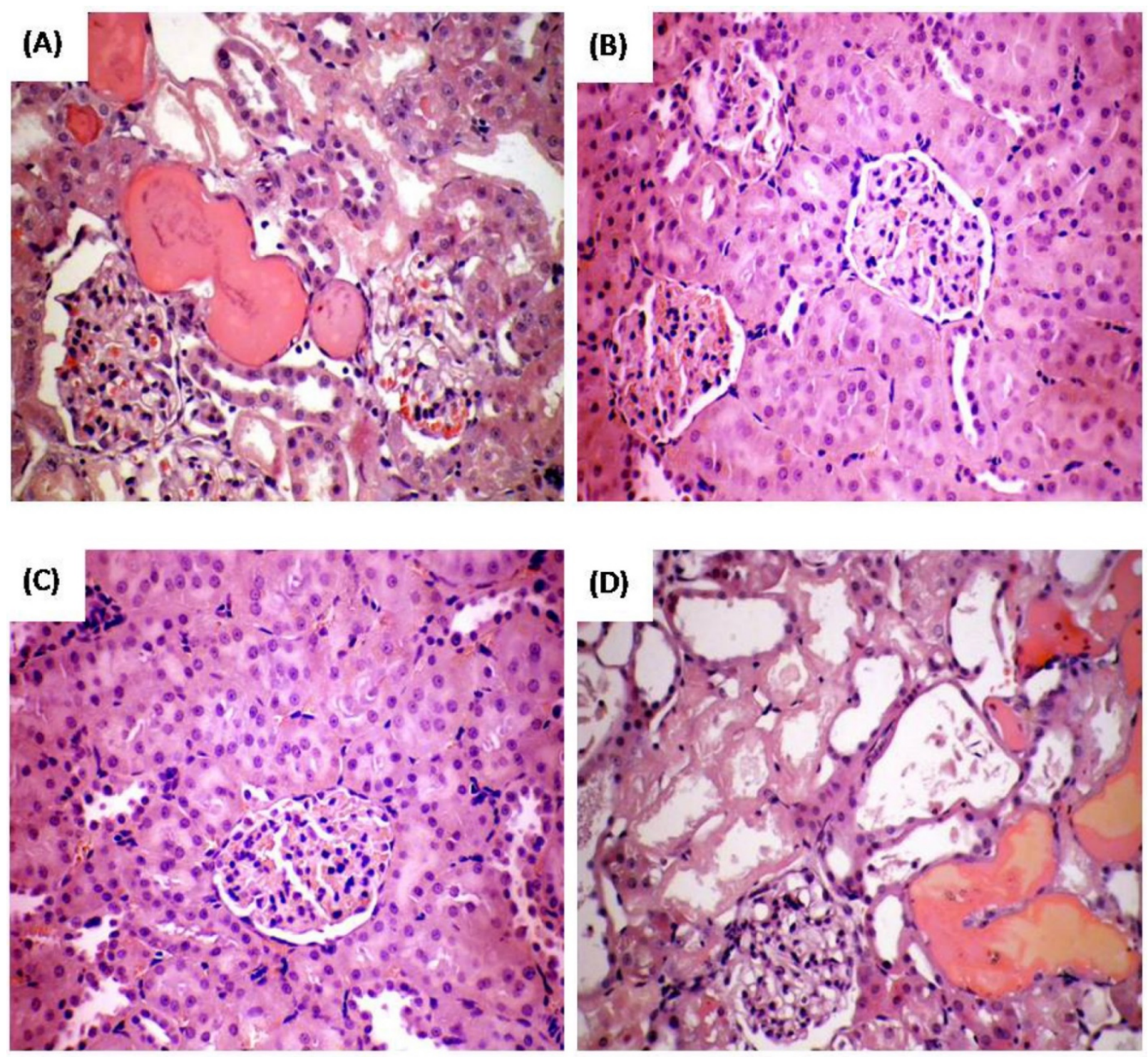

(E)

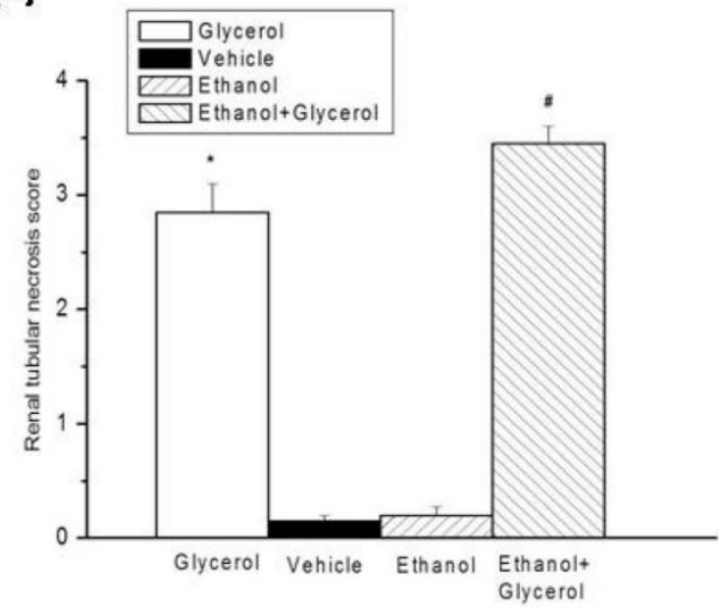

Figure 2. Histopathologic changes of kidneys after rhabdomyolysis-induced acute renal failure in rats. Histological sections from the Glycerol group (A), Vehicle group (B), Ethanol group (C), and Ethanol + Glycerol group $(\mathbf{D})$, stained with hematoxylin and eosin (magnification $\times 200$ ). Renal tissue injury score after rhabdomyolysis-induced acute renal failure in rats $(\mathbf{E}) .{ }^{*} p<0.05$ for the Glycerol group compared with the Vehicle group. \#p $<0.05$ for the Ethanol + Glycerol group compared with the Glycerol group. 

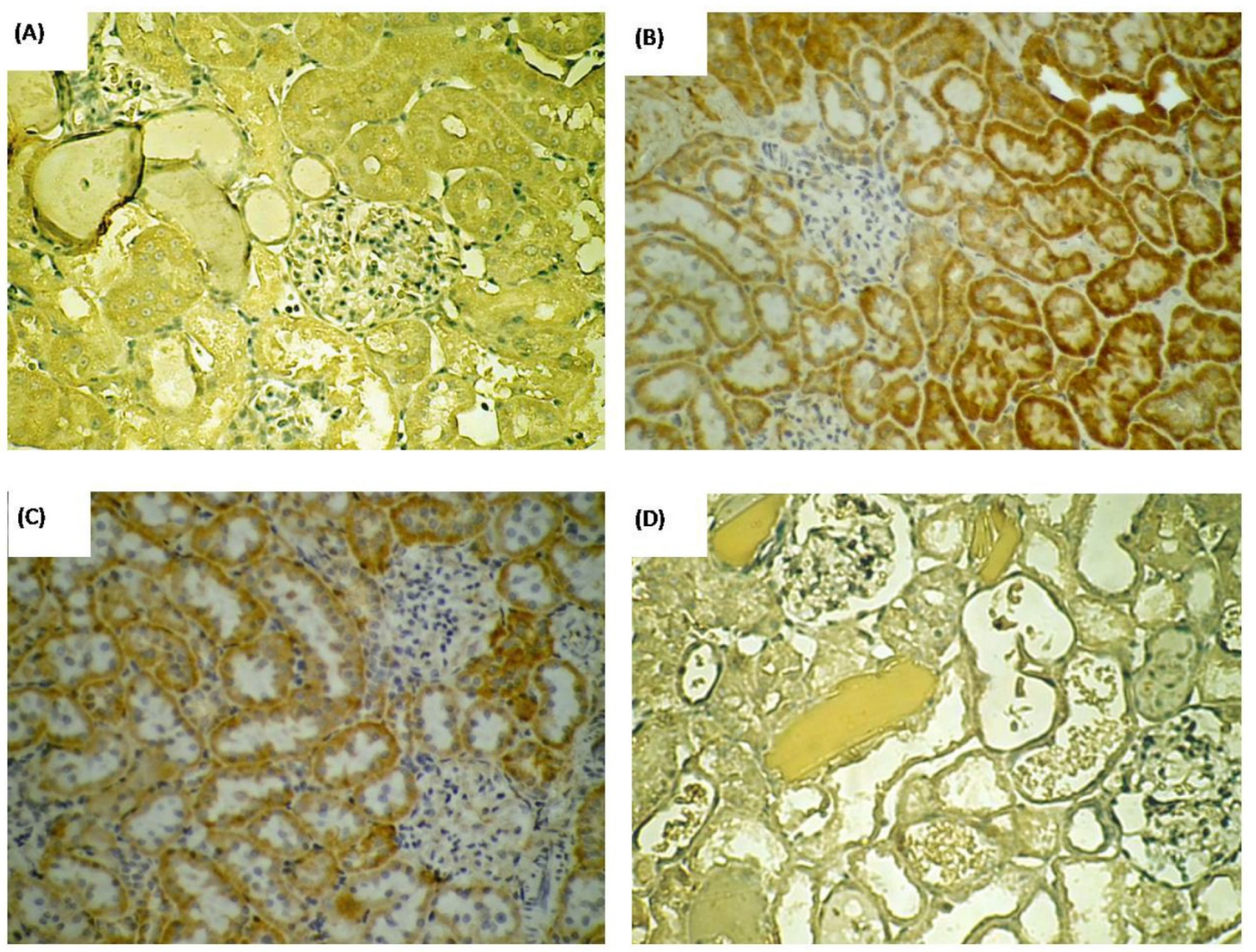

(E)

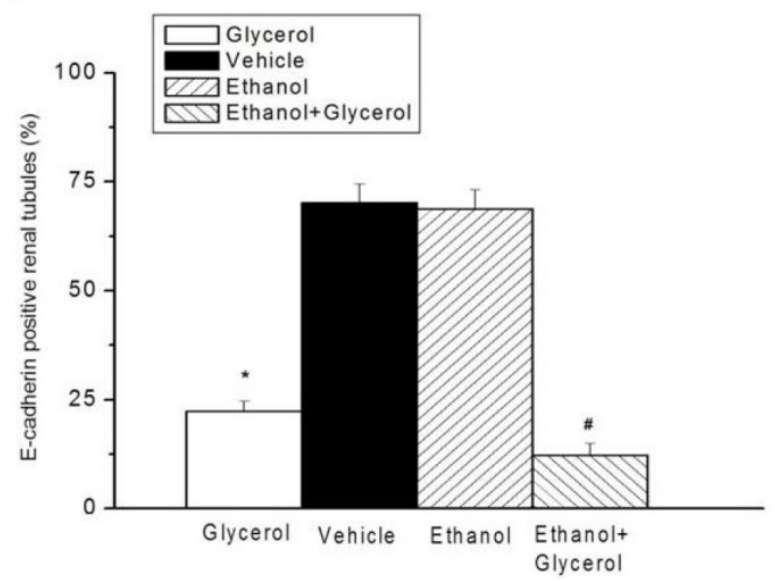

Figure 3. Immunohistochemical staining for E-cadherin after rhabdomyolysis-induced acute renal failure in rats. Histological sections from the Glycerol group (A), Vehicle group (B), Ethanol group (C), and Ethanol + Glycerol group (D) (magnification $\times 200)$. Renal tubular E-cadherin positive scores after rhabdomyolysis-induced acute renal failure in rats $(\mathbf{E})$. ${ }^{*} p<0.05$ for the Glycerol group compared with the Vehicle group. ${ }^{\#} p<0.05$ for the Ethanol + Glycerol group compared with the Glycerol group. 

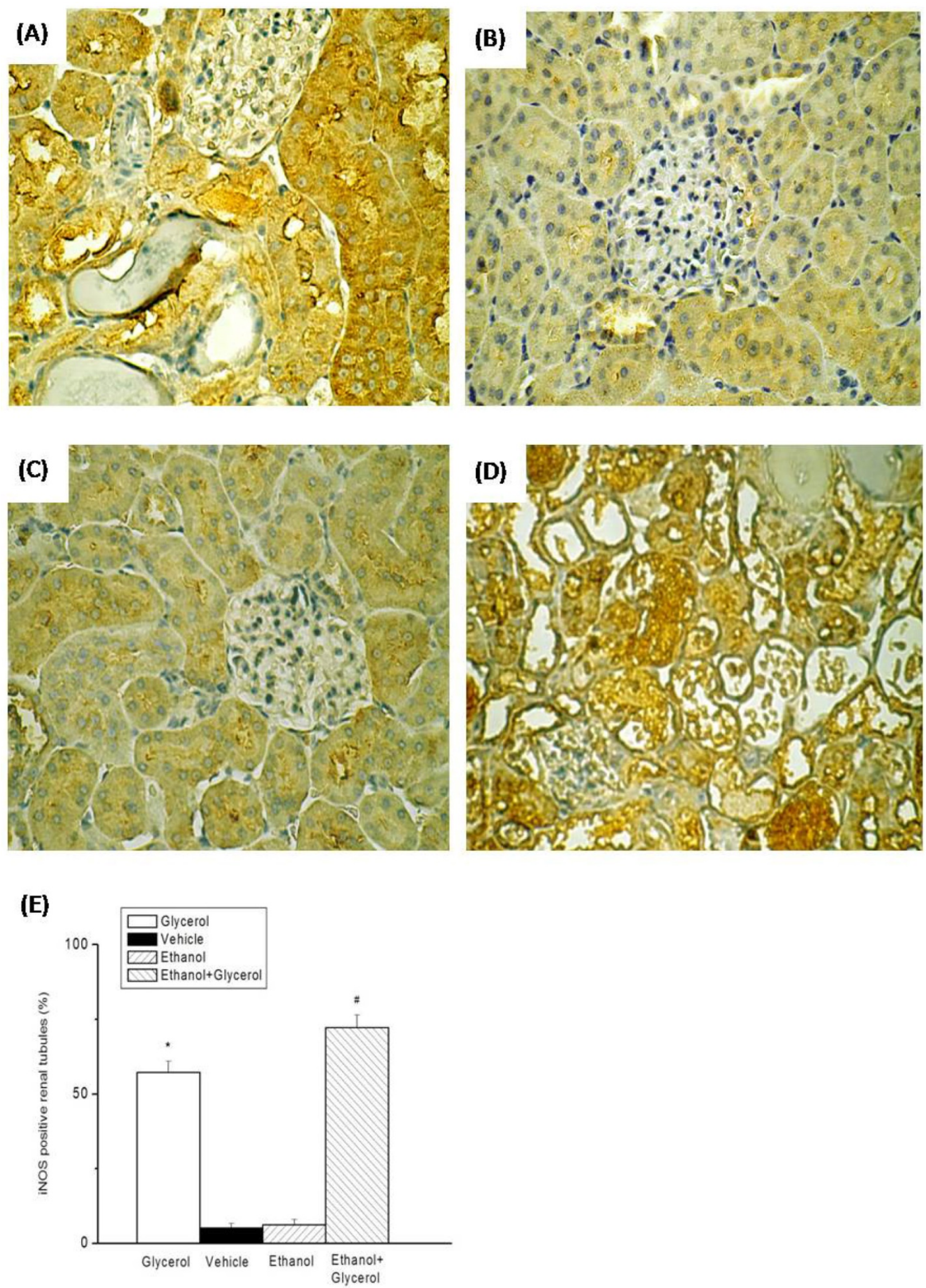

Figure 4. Immunohistochemical staining of iNOS after rhabdomyolysis-induced acute renal failure in rats. Histological sections from the Glycerol group (A), Vehicle group (B), Ethanol group (C), and Ethanol + Glycerol group (D) (magnification $\times 200$ ). Renal tubular iNOS scores after rhabdomyolysis-induced acute renal failure in rats $(\mathbf{E}) .{ }^{*} p<0.05$ for the Glycerol group compared with the Vehicle group. $\# p<0.05$ for the Ethanol + Glycerol group compared with the Glycerol group. 

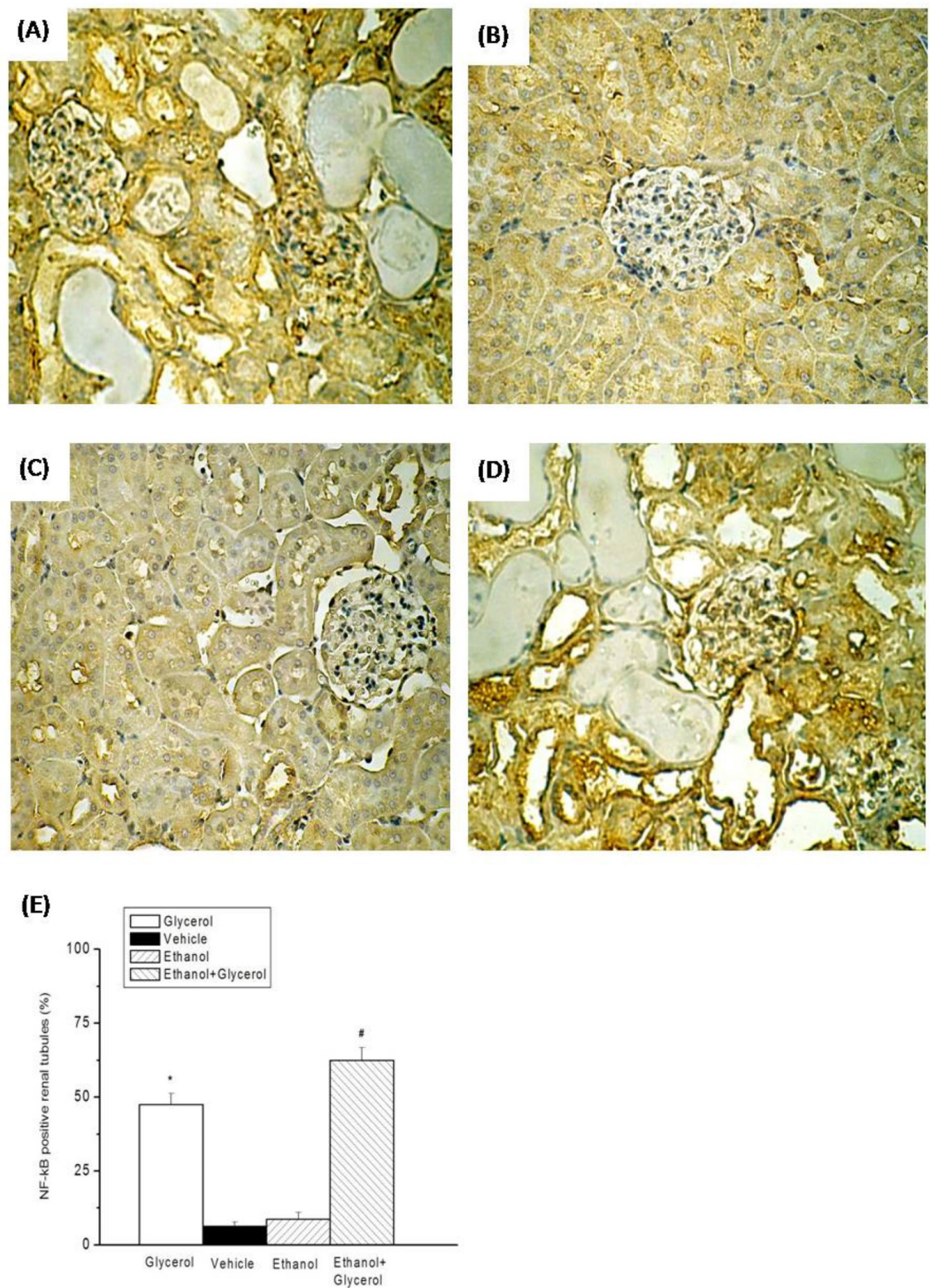

Figure 5. Immunohistochemical staining for NF-KB after rhabdomyolysis-induced acute renal failure in rats. Histological sections from the Glycerol group (A), Vehicle group (B), Ethanol group (C), and Ethanol + Glycerol group (D) (magnification $\times 200$ ). Renal tubular NF-KB positive scores after rhabdomyolysis-induced acute renal failure in rats $(\mathbf{E})$. ${ }^{*} p<0.05$ for the Glycerol group compared with the Vehicle group. ${ }^{\#} p<0.05$ for the Ethanol + Glycerol group compared with the Glycerol group. 
There are several mechanisms of rhabdomyolysis-induced ARF, such as hypovolemia, intraluminal obstruction by cast formation, induction of inflammation, generation of free radicals, scavenging of nitric oxide, and activation of the endothelin receptor, and these have a synergistic effect on renal vasoconstriction, and a direct toxic effect on renal tubular cells [5, 7, 8, 21]. Following these acute renal tubular injuries, nitric oxide, intracellular calcium influx, and ROS disrupt mitochondrial function [22, 23]. Additionally, extracellular fluid leakage into the damaged muscles indirectly activates the renin-angiotensin-aldosterone system and myoglobin-induced nitric oxide scavenging, and the release of cytokines leads to renal vasoconstriction [21, 24]. These immune-stimulatory molecules can activate infiltrated macrophages and lymphocytes to produce complement factors, Toll-like receptors, and NF- $\mathrm{kB}$, thereby promoting the release of pro-inflammatory cytokines [25]. Moreover, the loss of cell adhesion due to reduced levels of E-cadherin weakens the junctions between cells, allowing filtrate to leak back into the renal interstium, which impairs renal function [22]. According to previous studies, renal tubular epithelial cells were considered to undergo epithelial-mesenchymal transition (EMT) with the expression of fibroblast markers to become matrix-producing fibroblasts, and therefore contribute to renal fibrosis in chronic kidney diseases [26]. During the phenotype conversion, there is an induction of mesenchymal markers and disappearance of epithelial markers of epithelial cells, like E-cadherin, which is essential for the structural integrity of renal epithelium [26]. Tubular epithelial cells which manifested EMT, could be induced by various profibrotic cytokines, particularly TGF- $\beta 1$, and be associated with chronic interstitial inflammation that could be an adaptive response of epithelial cells to a changing microenvironment [27, 28]. Studies had shown that inflammation was considered to be important in the initiation of renal fibrosis, and non-resolving inflammation after chronic injury is a relentless driver of fibrogenesis because it creates a vicious cycle to tissue damage and fibrosis [29]. There is also increased expression of iNOS, which counteracts the responses elicited by angiotensin II and endothelin, and maintains blood flow by offsetting their vasoconstrictive effects [23]. Previous research reported that addition of myoglobin to renal tubules increased oxidative stress and mitochondrial dysfunction due to uncoupling of oxidative phosphorylation and increased nitric oxide synthesis [30]. In the present study, we found that glycerol-induced rhabdomyolysis alone increased renal tubular expression of iNOS and NF-KB and decreased expression of E-cadherin. This indicates a probable role of inflammation and oxidative stress in the pathogenesis of ARF.

Excessive alcohol consumption correlates with several diseases, particularly liver diseases, but also with coronary artery disease and hypertension [31, 32]. Less is known about the relationship between alcohol consumption and kidney disease, and there are some controversial results [33]. The 5-year observational AusDiab study examined 6529 adults and found that individuals who were moderate drinkers (10-30 g/day) or heavy drinkers (> $30 \mathrm{~g} /$ day) had a higher risk for albuminuria, a predictor of accelerated loss of kidney function [33]. Van Thiel et al. found that compared to iso-caloric controls, alcohol-fed rats had significantly reduced renal function and structural damage of the kidneys, including cellular and mitochondrial swelling in the proximal renal tubules, cellular necrosis in the loops of Henle, luminal dilation, focal cell loss and cellular necrosis in distal renal tubules, and edematous and fibrotic changes in the interstitium [14]. These findings, in agreement with other research and the results of the present study, indicate that heavy alcohol drinking has a direct nephrotoxic effect [14, 33, 34].

Previous research indicated that feeding rats a large amount of alcohol led to significantly increased activities of malondialdehyde, superoxide dismutase, and catalase, atrophic renal corpuscles, and dilation and congestion of the peritubular vessels [35]. Binge drinking can cause ARF due to rhabdomyolysis either directly, because ethanol has a toxic effect on skeletal muscles [36], or indirectly, because it leads to volume depletion due to diuresis, and then vasoconstriction, and acceleration of renal dysfunction via disturbance of systemic and intrarenal vasoactive factors that maintain renal hemodynamics [34]. We found that co-administration of ethanol with glycerol aggravated the harmful effects of glycerol-induced rhabdomyolysis, in that it aggravated kidney damage as indicated by increased renal tubular necrosis scores, increased renal tubular expression of iNOS and NF-kB, and decreased expression of E-cadherin. This implicates inflammation and oxidative stress in the pathogenesis of kidney damage in the presence of rhabdomyolysis. In agreement, there is evidence that agents which target inflammatory and pro-oxidant pathways, such as mannitol, N-acetylcysteine, vitamin E, flavonoids, L-carnitine, and pentoxifylline, can effectively treat rhabdomyolysis, in addition to traditional methods [7, 9, 37]. Taken together, our results suggest that acute alcohol intoxication exacerbates rhabdomyolysis-induced renal damage, due to its induction of vasoconstriction, renal tubular 
inflammation, and oxidation-related injuries.

\section{Conclusion}

In conclusion, our study of a rat model of rhabdomyolysis indicates that acute ethanol intoxication exacerbates the effects of rhabdomyolysis on muscular, hepatic, and renal function, further increases renal tubular expression of NF-kB, iNOS, and further decreases the expression of E-cadherin. These results support our hypothesis that acute alcohol consumption has harmful synergistic effects on rhabdomyolysis-induced organ damage, especially ARF.

\section{Acknowledgments}

This work was supported in part by a grant from the Tzu Chi University (TCIRP 98004-02).

\section{Competing Interests}

The authors have declared that no competing interest exists.

\section{References}

1. Bosch X, Poch E, Grau JM. Rhabdomyolysis and acute kidney injury. N Engl J Med. 2009; 361: 62-72.

2. Giannoglou GD, Chatzizisis YS, Misirli G. The syndrome of rhabdomyolysis: Pathophysiology and diagnosis. Eur J Intern Med. 2007; 18: 90-100.

3. Khan FY. Rhabdomyolysis: a review of the literature. Neth J Med. 2009; 67: 272-83.

4. Grossman RA, Hamilton RW, Morse BM, Penn AS, Goldberg M. Nontraumatic rhabdomyolysis and acute renal failure. N Engl J Med. 1974; 291: 807-11.

5. Zager RA, Johnson AC, Becker K. Plasma and urinary heme oxygenase-1 in AKI. J Am Soc Nephrol. 2012; 23: 1048-57.

6. Zager RA, Foerder CA. Effects of inorganic iron and myoglobin on in vitro proximal tubular lipid peroxidation and cytotoxicity. J Clin Invest. 1992; 89: 989-95.

7. Huerta-Alardin AL, Varon J, Marik PE. Bench-to-bedside review: Rhabdomyolysis -- an overview for clinicians. Crit Care. 2005; 9: 158-69.

8. Vanholder R, Sever MS, Erek E, Lameire N. Rhabdomyolysis. J Am Soc Nephrol. 2000; 11: 1553-61.

9. Panizo N, Rubio-Navarro A, Amaro-Villalobos JM, Egido J, Moreno JA. Molecular Mechanisms and Novel Therapeutic Approaches to Rhabdomyolysis-Induced Acute Kidney Injury. Kidney Blood Press Res. 2015; 40: 520-32.

10. Szabo G, Mandrekar P. A recent perspective on alcohol, immunity, and host defense. Alcohol Clin Exp Res. 2009; 33: 220-32.

11. Hu TM, Lee RP, Lee CJ, Subeq YM, Lin NT, Hsu BG. Heavy ethanol intoxication increases proinflammatory cytokines and aggravates hemorrhagic shock-induced organ damage in rats. Mediators Inflamm. 2013; 2013: 121786.

12. Tanaka A, Cui R, Kitamura A, Liu K, Imano H, Yamagishi $K$, et al. Heavy Alcohol Consumption is Associated with Impaired Endothelial Function. I Atheroscler Thromb. 2016; 23: 1047-54.

13. Caimi G, Carollo C, Lo Presti R. Chronic renal failure: oxidative stress, endothelial dysfunction and wine. Clin Nephrol. 2004; 62: 331-5.

14. Van Thiel DH, Gavaler JS, Little JM, Lester R. Alcohol: its effect on the kidney. Metabolism. 1977; 26: 857-66.

15. Amanvermez R, Demir S, Tuncel OK, Alvur M, Agar E. Alcohol-induced oxidative stress and reduction in oxidation by ascorbate/L-cys/ L-met in the testis, ovary, kidney, and lung of rat. Adv Ther. 2005; 22: 548-58.

16. Yang FL, Subeq YM, Chiu YH, Lee RP, Lee CJ, Hsu BG. Recombinant human erythropoietin reduces rhabdomyolysis-induced acute renal failure in rats. Injury. 2012; 43: 367-73

17. Subeq YM, Wu WT, Lee CJ, Lee RP, Yang FL, Hsu BG. Pentobarbital reduces rhabdomyolysis-induced acute renal failure in conscious rats. J Trauma. 2009; 67: 132-8.

18. Sauret JM, Marinides G, Wang GK. Rhabdomyolysis. Am Fam Physician. 2002; 65: 907-12.

19. Knochel JP. Mechanisms of rhabdomyolysis. Curr Opin Rheumatol. 1993; 5: 725-31.
20. Ward MM. Factors predictive of acute renal failure in rhabdomyolysis. Arch Intern Med. 1988; 148: 1553-7.

21. Krouzecky A, Matejovic M, Rokyta R, Jr., Novak I. [Rhabdomyolysis--development, causes, sequelae and therapy]. Vnitr Lek. 2003; 49: 668-72.

22. Prozialeck WC, Edwards JR. Cell adhesion molecules in chemically-induced renal injury. Pharmacol Ther. 2007; 114: 74-93.

23. Aksu U, Demirci C, Ince C. The pathogenesis of acute kidney injury and the toxic triangle of oxygen, reactive oxygen species and nitric oxide. Contrib Nephrol. 2011; 174: 119-28.

24. Blomberg LM, Blomberg MR, Siegbahn PE. A theoretical study of myoglobin working as a nitric oxide scavenger. J Biol Inorg Chem. 2004; 9: 923-35.

25. Gonzalez-Michaca L, Farrugia G, Croatt AJ, Alam J, Nath KA. Heme: a determinant of life and death in renal tubular epithelial cells. Am J Physiol Renal Physiol. 2004; 286: F370-7.

26. Liu Y. Cellular and molecular mechanisms of renal fibrosis. Nat Rev Nephrol. 2011; 7: 684-96.

27. Neilson EG. Mechanisms of disease: Fibroblasts--a new look at an old problem. Nat Clin Pract Nephrol. 2006; 2: 101-8.

28. Yang J, Liu Y. Dissection of key events in tubular epithelial to myofibroblast transition and its implications in renal interstitial fibrosis. Am J Pathol. 2001; 159: 1465-75.

29. Liu $Y$. New insights into epithelial-mesenchymal transition in kidney fibrosis. J Am Soc Nephrol. 2010; 21: 212-22.

30. Plotnikov EY, Chupyrkina AA, Pevzner IB, Isaev NK, Zorov DB. Myoglobin causes oxidative stress, increase of NO production and dysfunction of kidney's mitochondria. Biochim Biophys Acta. 2009; 1792: 796-803.

31. Corrao G, Rubbiati L, Bagnardi V, Zambon A, Poikolainen K. Alcohol and coronary heart disease: a meta-analysis. Addiction. 2000; 95: 1505-23.

32. Taylor B, Irving HM, Baliunas D, Roerecke M, Patra J, Mohapatra S, et al. Alcohol and hypertension: gender differences in dose-response relationships determined through systematic review and meta-analysis. Addiction. 2009; 104: 1981-90.

33. White SL, Polkinghorne KR, Cass A, Shaw JE, Atkins RC, Chadban SJ. Alcohol consumption and 5-year onset of chronic kidney disease: the AusDiab study. Nephrol Dial Transplant. 2009; 24: 2464-72.

34. Tsuboi N, Yoshida H, Shibamura K, Hikita M, Tomonari H, Kuriyama S, et al. Acute renal failure after binge drinking of alcohol and nonsteroidal antiinflammatory drug ingestion. Intern Med. 1997; 36: 102-6.

35. Sonmez MF, Narin F, Akkus D, Turkmen AB. Melatonin and vitamin C ameliorate alcohol-induced oxidative stress and eNOS expression in rat kidney. Ren Fail. 2012; 34: 480-6.

36. Hewitt SM, Winter RJ. Rhabdomyolysis following acute alcohol intoxication. J Accid Emerg Med. 1995; 12: 143-4.

37. Malik GH. Rhabdomyolysis and Myoglobin-induced Acute Renal Failure. Saudi J Kidney Dis Transpl. 1998; 9: 273-84. 